\title{
Thermodynamic approach to rheological modeling and simulations at the configuration space level of description
}

\author{
R. J. J. Jongschaap, ${ }^{\text {a) }}$ A. I. M. Denneman, and W. Conrads \\ Rheology Group, Faculty of Applied Physics, University of Twente, \\ P.O. Box 217, 7500 AE Enschede, The Netherlands
}

(Received 29 May 1996; final revision received 25 October 1996)

\begin{abstract}
Synopsis
The so-called matrix model is a general thermodynamic framework for microrheological modeling. This model has already been proven to be applicable for a wide class of systems, in particular to models formulated at the configuration tensor level of description. For models formulated at the configuration space level of description a matrix formulation is readily obtained, but for the subsequent analysis one still needs an explicit solution of the configuration space distribution functions. In the present paper we describe an approach in which this problem is solved by combining the matrix model with a Lagrangian simulation method in configuration space developed recently by Szeri and Leal. The result is a consistent and unified formulation of stress tensor expressions, including the stress averaging, and the evolution equations. This formulation is also suited for numerical simulations. In this way, the range of applicability of the matrix model is extended substantially. In order to clarify the principles of the method and some aspects of its implementation, a simple example is discussed in some detail. (C) 1997 The Society of Rheology.
\end{abstract} [S0148-6055(97)00402-1]

\section{INTRODUCTION}

A phenomenological description of the rheological properties of a material consists of the specification of an equation relating the stress tensor to the deformation (history) of the material: the so-called constitutive equation. For complex systems an adequate formulation of this kind is, however, often not available and even if such a macroscopic constitutive equation exists it is not always obvious how it is related to the underlying phenomena on a microscopic scale. For that reason a treatment based upon molecular modeling is often preferable.

In the present paper we follow an approach that combines the advantages of the continuum as well as the microscopic approach. The key to this is a treatment at a level of description inbetween the phenomenological and the molecular level, supplemented by additional information derived from thermodynamic considerations. To this end, we use a formulation called the matrix model, which was derived earlier from thermodynamic principles [Jongschaap (1990)] and has proved to be applicable for a wide class of systems [Jongschaap et al. (1994)].

Advantages of this formulation are that (i) it provides a consistent framework for the treatment of various rheological models at several levels of description, (ii) it is consis-

\footnotetext{
${ }^{\text {a) }}$ Corresponding author.
} 
tent with classical nonequilibrium thermodynamics [Groot and Mazur (1969)], in particular with approaches using specified [Kluitenberg (1962); Leonov (1976)] or unspecified [Meixner (1953)] internal variables, (iii) it is consistent with the Hamiltonian-bracket formulation of rheological models developed by Grmela [Grmela (1986)] and Beris and Edwards [Beris and Edwards (1994)], (iv) it provides a simple and elegant derivation of consistency relations of stress tensor and evolution equations in accordance with results obtained by Grmela [Grmela (1986)] and that (v) it uses a mathematical (matrix) formulation that is a compact, complete, and accurate representation of the essential thermodynamic and structural properties.

On the one hand, the matrix model has proven to be applicable to many models at the so-called structure or configuration tensor level of description and has offered new insight to particular aspects of these models. For instance, the necessity of a fore factor in the stress tensor if nonaffine convection is assumed as was noted earlier by Larson [Larson (1984)] and the result that the nondiagonal elements in the Onsager matrix in a model by Leonov [Leonov (1976)] are zero, etc. The model was also used with success by others, for instance by Schieber [Schieber and Öttinger (1994)] in a study of stress tensor expressions in systems with internal viscosity and by Tervoort [Tervoort (1996)] in a study of the behavior of glassy polymers.

On the other hand, the application to models based upon a description with configuration space distribution functions (i.e., the configuration space level of description) was still not satisfactory. The relevant equations of the models at the latter level of description may readily be written in the required matrix form (see the examples given in Sec. II B below), but an expression for the configuration space distribution function is still needed in this case in order to calculate thermodynamic forces and to perform the necessary averaging.

In the present paper this problem is solved by using a Lagrangian formulation in configuration space that is essentially the so-called double-Lagrangian technique, proposed recently by Szeri and Leal [Szeri and Leal (1992)]. In Sec. III it will be shown that this method is fully compatible with our matrix representation and that by combining both methods not only a consistent formulation of stress tensor and evolution equations is achieved but also the stress averaging is included in the matrix formulation. This treatment is suited for computer simulations and offers promising challenges for the treatment of complex fluids. In the present paper, however, we will restrict ourselves to the principles of the method and in Sec. IV we will, therefore, discuss only a simple case of a simulation of this type. This example indicates that an efficient implementation of the method should be possible. Moreover, an optimized Lagrangian description provides an alternative set of thermodynamic state variables for the system.

\section{MATRIX FORMULATION OF STRESS TENSOR AND EVOLUTION EQUATIONS}

In Jongschaap (1990) a generalized formulation of constitutive equations was proposed, that allows a consistent formulation of stress tensor and evolution expressions of rheological models at various levels of description. This formulation called the matrix model, is based on thermodynamic principles. On one hand it uses the concept of state variables that allows the expression of the rate of change of the internal energy $U$ as

$$
\dot{U}=T \dot{S}+F \dot{X},
$$

in which $T$ is the temperature, $S$ the entropy, $F$ a (set of) thermodynamic force(s): $F=\partial U / \partial X$ and $X$ a (set of) thermodynamic variable(s) of state. On the other hand, the 
internal energy $U$ is related to the external forces $F^{e}$ and external rate variables $\dot{X}^{e}$ through the balance equation

$$
\dot{U}=\dot{Q}+F^{e} \dot{X}^{e},
$$

in which $\dot{Q}$ is a heat flux. This balance equation describes the coupling of the system with its environment. The dissipative and nondissipative couplings between the variables $F^{e}, \dot{X}^{e}, \dot{X}$, and $F$, which may have a specific tensorial character, can be specified then as [Jongschaap (1990), Jongschaap (1991)]

$$
\left(\begin{array}{c}
F^{e} \\
\dot{X}
\end{array}\right)=\left(\begin{array}{cc}
\eta & -\Lambda^{T} \\
\Lambda & \beta
\end{array}\right) \cdot\left(\begin{array}{l}
\dot{X}^{e} \\
-F
\end{array}\right),
$$

in which the superscript $T$ denotes a transposed tensor, defined in accordance with the tensorial degree of the element $\Lambda$. The symmetry properties of the matrix, which imply the consistency of the force and the evolution equations contained in the matrix representation (3), are derived from a principle called Macroscopic Time Reversal [Jongschaap (1990)], which is essentially a statement about the parity of variables under a change of sign of the external rate variable $\dot{X}^{e}$. With this principle it is possible to obtain in a relatively simple way earlier results derived by more sophisticated statistical mechanical considerations by Grmela [Grmela (1986)]. The results are also in accordance with similar ideas of time reversal introduced by Martin [Martin et al. (1972)] and used by Lhuillier [Lhuillier and Ouibrahim (1980)], Maugin [Maugin and Drouot (1983)] and others. The contributions associated with the matrix elements $\eta$ and $\beta$ turn out to have a dissipative and the one's associated with $\Lambda$ a reversible character.

\section{A. The configuration tensor level of description}

At the configuration tensor level of description the thermodynamic variable of state is a configuration tensor $\mathbf{S}$. The internal energy is given at this level by a fundamental equation $U=U(S, \mathbf{S})$ in which $\mathbf{S}$ is the entropy and the free energy $A=U-T S$ by a fundamental equation $A=A(T, \mathbf{S})$ as function of the temperature $T$ and $\mathbf{S}$. As in Eq. (1) we express the rate of change of $U$ as

$$
\dot{U}=T \dot{S}+\mathbf{M}: \dot{\mathbf{S}}
$$

in which $\mathbf{M}$ is the thermodynamic force,

$$
\mathbf{M}=\frac{\partial A}{\partial \mathbf{S}} .
$$

The balance equation of power similar to Eq. (2) becomes

$$
\dot{U}=\dot{Q}+\mathbf{T}: \mathbf{L},
$$

in which $\mathbf{T}$ is the stress tensor and $\mathbf{L}$ the velocity gradient tensor. The matrix formulation similar to Eq. (3) becomes

$$
\left(\begin{array}{c}
\mathbf{T} \\
\dot{\mathbf{S}}
\end{array}\right)=\left(\begin{array}{cc}
\eta & -\Lambda^{T} \\
\Lambda & \beta
\end{array}\right):\left(\begin{array}{c}
\mathbf{L} \\
-\mathbf{M}
\end{array}\right) .
$$

The matrix elements $\eta, \Lambda$, and $\beta$ are fourth-order tensors and $\Lambda^{T}$ is defined, in this case, by $\Lambda_{i j k m}^{T}=\Lambda_{k m i j}$. 
In Jongschaap et al. (1994) it is shown that the representation (7) applies to many existing rheological models, like the Maxwell-Giesekus [Giesekus (1982b); Giesekus (1982a)] and Leonov [Leonov (1976)] models. In the next section some results for the case of the elastic dumbbell model are summarized.

\section{Example: Elastic dumbbell}

For the elastic dumbbell model an appropriate choice of the configuration tensor $\mathbf{S}$ is the second moment $\mathbf{S}=\langle\mathbf{q q}\rangle$ of the distribution function $\psi(\mathbf{q}, t)$ of the end-to-end vector q. The free-energy function $A(T, S)$ then becomes

$$
A=\frac{1}{2} n \kappa \operatorname{tr} \mathbf{S}-\frac{1}{2} n k T \ln \operatorname{det} \mathbf{S},
$$

with $\kappa$ an elastic modulus and $n$ the number density of dumbbells. The stress tensor is given by expression

$$
\mathbf{T}=n(\kappa \mathbf{S}-k T \mathbf{1}),
$$

and the evolution equation of $\mathbf{S}$ reads

$$
\dot{\mathbf{S}}=\mathbf{L} \cdot \mathbf{S}+\mathbf{S} \cdot \mathbf{L}^{T}+\frac{4 k T}{\zeta}\left(\mathbf{1}-\frac{\kappa}{k T} \mathbf{S}\right)
$$

The matrix representation of these equations is of the form (7) with $\eta=0$,

$$
\Lambda_{i j k m}=\delta_{i k} S_{m j}+S_{i m} \delta_{j k},
$$

and

$$
\beta=\frac{4}{n \zeta} \Lambda,
$$

in which $\zeta$ is a hydrodynamic friction coefficient.

For nonlinear dumbbells, a closed description at the configuration tensor level is in general not possible. In that case, a description at the configuration space level is needed. This will be discussed in Sec. III A.

\section{B. The configuration space level of description}

At the configuration space level of description, the thermodynamic state of a system is described by the time dependent probability density $\psi(X, t)$ of a (set of) configurational variable(s) $X$. Instead of Eq. (1) the rate of change of internal energy is expressed as

$$
\dot{U}=T \dot{S}+\int \mu(X) \frac{\partial \psi}{\partial t} d X
$$

with $T$ the temperature, $S$ the entropy, and $\mu(X)$ a chemical potential in configuration space. For a discussion of the evolution equations for $\psi(X, t)$, we refer to the Appendix.

Substitution of the equation of continuity

$$
\frac{\partial \psi}{\partial t}=-\frac{\partial}{\partial X} \cdot(\dot{X} \psi)
$$

in Eq. (13) and integration by parts gives 


$$
\dot{U}=T \dot{S}-\int \mu \frac{\partial}{\partial X} \cdot(\psi \dot{X}) d X=T \dot{S}+\int \psi \frac{\partial \mu}{\partial X} \cdot \dot{X} d X=T \dot{S}+\int \psi F(X) \cdot \dot{X} d X
$$

with the thermodynamic force

$$
F(X)=\frac{\partial \mu}{\partial X}
$$

At this level of description the balance equation of power similar to Eq. (2) is again given by Eq. (6) where the macroscopic stress tensor $\mathbf{T}$ is treated as the average of a microscopic force dipole $\tau$

$$
\mathbf{T}=n \int \psi \tau d X
$$

We now list the associated matrix representations for some well-known models [Jongschaap (1991)]

(1) Elastic dumbbell:

$$
\left(\begin{array}{c}
\tau \\
\dot{\mathbf{q}}
\end{array}\right)=\left(\begin{array}{cc}
\mathbf{0 :} & -(\mathbf{1 q})^{T} \cdot \\
\mathbf{1 q}: & \frac{2}{\zeta} \mathbf{1} .
\end{array}\right)\left(\begin{array}{c}
\mathbf{L} \\
-\frac{\partial \mu}{\partial \mathbf{q}}
\end{array}\right) .
$$

In this case,

$$
\mu=k T \ln \frac{\psi}{\psi_{0}},
$$

in which $\psi_{0}$ is the equilibrium distribution and the thermodynamic force $\mathbf{f}=\partial \mu / \partial \mathbf{q}$ may be considered as the sum of minus the Brownian force $\mathbf{f}^{B}=-k T(\partial / \partial \mathbf{q}) \ln \psi$ and the connector force $\mathbf{f}^{c}=-k T(\partial / \partial \mathbf{q}) \ln \psi_{0}$.

(2) Rigid dumbbell:

$$
\left(\begin{array}{c}
\tau \\
\dot{\mathbf{e}}
\end{array}\right)=\left(\begin{array}{cc}
\frac{1}{2} \zeta q^{2} \text { eeee: } & -(\mathbf{P e})^{T} \cdot \\
\mathbf{P e}: & \frac{2}{\zeta} \mathbf{1} .
\end{array}\right)\left(\begin{array}{c}
\mathbf{L} \\
-\frac{\partial \mu}{\partial \mathbf{e}}
\end{array}\right) .
$$

In these expressions $\mathbf{P}$ is the projection operator $\mathbf{P}=\mathbf{1}-\mathbf{e e}, \mathbf{e}$ a unit vector in the direction of the dumbbell, and

$$
\mu=k T \ln \psi
$$

(3) Nematic liquid crystals [Doi (1981)]:

$$
\left(\begin{array}{c}
\tau \\
\dot{\mathbf{e}}
\end{array}\right)=\left(\begin{array}{cc}
\mathbf{0}: & -(\mathbf{P e})^{T} . \\
\mathbf{P e}: & \frac{\bar{D}_{r}}{k T} \mathbf{1}
\end{array}\right)\left(\begin{array}{c}
\mathbf{L} \\
-\frac{\partial \mu}{\partial \mathbf{e}}
\end{array}\right),
$$

in which $\bar{D}_{r}$ is an average rotary diffusivity and

$$
\mu=k T \ln \psi+\Phi,
$$


with $\Phi=$ const $-\frac{3}{2} n U k T \mathbf{e e}:\langle\mathbf{e e}-\mathbf{1}\rangle$, the Mayer-Saupe potential. Here, $U$ is a parameter proportional to $b L^{2}$, in which $b$ is the diameter and $L$ the length of the rodlike particles.

(4) Reptation models:

The Doi and Edwards model

$$
\left(\begin{array}{c}
\tau \\
\dot{\mathbf{e}} \\
\dot{s}
\end{array}\right)=\left(\begin{array}{ccc}
\mathbf{0}: & -(\mathbf{P} e)^{T} . & \mathbf{0} \\
\mathbf{P e}: & \mathbf{0} & \mathbf{0} \\
\mathbf{0}: & \mathbf{0} & \frac{D}{k T}
\end{array}\right) \cdot\left(\begin{array}{c}
\mathbf{L} \\
-\frac{\partial \mu}{\partial \mathbf{e}} \\
-\frac{\partial \mu}{\partial s}
\end{array}\right) .
$$

The Curtiss-Bird [Curtiss and Bird (1981)], reptating rope [Jongschaap (1988)] model

$$
\left(\begin{array}{c}
\tau \\
\dot{\mathbf{e}} \\
\dot{s}
\end{array}\right)=\left(\begin{array}{ccc}
\frac{1}{2} \zeta s(L-s) \text { eeee: } & -(\mathbf{P e})^{T} . & \mathbf{0} \\
\mathbf{P e} & \mathbf{0} & \mathbf{0} \\
\mathbf{0 :} & \mathbf{0} & \frac{D}{k T}
\end{array}\right) \cdot\left(\begin{array}{c}
\mathbf{L} \\
-\frac{\partial \mu}{\partial \mathbf{e}} \\
-\frac{\partial \mu}{\partial s}
\end{array}\right) .
$$

In the cases (24) and (25) the state space consists of the orientation vectors $\mathbf{e}$ and the scalar $s$ that measures the curvilinear position along a tube of length $L . D$ is a diffusion coefficient and $\mu$ a chemical potential in state space given by

$$
\mu=k T \ln \psi(\mathbf{e}, s) .
$$

As stated in the Introduction, it still remains a problem that the above equations must be closed by the specification of a probability densities. In the next section a method is presented by which this problem is solved.

\section{THE LAGRANGIAN METHOD}

In this section we discuss the combination of our matrix representation and the simulation method developed by Szeri and Leal [Szeri and Leal (1992)], which is based upon a Lagrangian description of the probability flux in configuration space. In fact, Szeri and Leal consider a combined Lagrangian formulation of the flow in configuration space and in ordinary physical space and, therefore, called their method a Double Lagrangian Method; here we only consider the flow in configuration space, so we will refer to it as the Lagrangian Method.

The flux velocity $\dot{X}(X, t)$ in the equation of continuity (14) is a smooth vector field in configuration space. It is determined by an evolution equation with convective, diffusive, and reversible parts. Some examples of these equations are contained in the matrix formulations given in Sec. II B and a more general discussion is given in the Appendix. With this flux velocity we associate a mapping $X=\chi\left(X_{0}, t\right)$ defined by the differential equation

$$
\frac{\partial \chi\left(X_{0}, t\right)}{\partial t}=\dot{X}\left(\chi\left(X_{0}, t\right), t\right)
$$

with the initial condition $\chi\left(X_{0}, 0\right)=X_{0}$. This mapping may be interpreted as a coordinate map between the initial configuration of the system and the "deformed" configuration at time $t$. 
The gradient of this mapping is the configuration space tensor field

$$
G\left(X_{0}, t\right)=\frac{\partial \chi\left(X_{0}, t\right)}{\partial X_{0}}
$$

The Jacobian of the mapping $\chi\left(X_{0}, t\right)$

$$
J\left(X_{0}, t\right)=\operatorname{det}\left[G\left(X_{0}, t\right)\right],
$$

determines the change of an infinitesimal volume element in configuration space

$$
d \chi\left(X_{0}, t\right)=J\left(X_{0}, t\right) d X_{0} .
$$

For the probability density $\psi(X, t)$ with $X=\chi\left(X_{0}, t\right)$, this implies

$$
\psi(X, t)=\frac{\psi\left(X_{0}, 0\right)}{J\left(X_{0}, t\right)}
$$

Assuming that at $t=0$ the system is in equilibrium, $\psi\left(X_{0}, 0\right)=\psi_{0}\left(X_{0}\right)$, we have

$$
\psi(X, t)=\frac{\psi_{0}\left(X_{0}\right)}{J\left(X_{0}, t\right)} .
$$

The ensemble average of a state variable $K(X)$ may be expressed now as

$$
\langle K\rangle=\int \psi(X, t) K(X) d X=\int \psi_{0}\left(X_{0}\right) K^{*}\left(X_{0}, t\right) d X_{0},
$$

with $K^{*}\left(X_{0}, t\right)=K\left[\chi\left(X_{0}, t\right)\right]=K(X)$. This property makes the Lagrangian method useful for computer simulations: if the mapping $\chi\left(X_{0}, t\right)$ is determined, a configuration space average may be performed as an average with respect to the equilibrium distribution $\psi_{0}$. In practice, the averaging (33) becomes a summation over a finite set of initial points $\left\{X_{01}, X_{02}, \ldots, X_{0 N}\right\}$ in configuration space, so

$$
\langle K\rangle=\sum_{i=1}^{N} w_{i} K\left[\chi\left(X_{0 i}, t\right)\right],
$$

with weight factors $w_{i}$ depending upon the distribution function $\psi_{0}$ and the discretization $\Delta X_{0 i}$,

$$
w_{i}=\frac{\psi_{0}\left(X_{0 i}\right) \Delta X_{0 i}}{\sum_{i=1}^{N} \psi_{0}\left(X_{0 i}\right) \Delta X_{0 i}}
$$

By associating points $X_{i}=\chi\left(X_{0 i}, t\right)$ with the points $X_{0 i}$ and taking into account all initial points $\left\{X_{0 i}\right\}$, one automatically samples at any time the relevant parts of configuration space. This implies a self-adaptive algorithm [Szeri and Leal (1992)].

A matrix formulation similar to Eq. (3) may be obtained by writing Eq. (15) in the form of (1) as $\dot{U}=T \dot{S}+\sum w_{i} F_{i} \dot{X}_{i}$ in which

$$
F_{i}=F\left(X_{i}, t\right)=\frac{\partial \mu\left(X_{i}, t\right)}{\partial X_{i}},
$$


with $X_{i}=\chi\left(X_{i 0}, t\right)$. The result is

$$
\left(\begin{array}{c}
F^{e} \\
\dot{X}_{1} \\
\dot{X}_{2} \\
\vdots \\
\dot{X}_{N}
\end{array}\right)=\left(\begin{array}{ccccc}
\eta & -\Lambda_{1}^{T} & -\Lambda_{2}^{T} & \cdots & -\Lambda_{3}^{T} \\
\Lambda_{1} & \beta_{1} & 0 & \cdots & 0 \\
\Lambda_{2} & 0 & \beta_{2} & \cdots & 0 \\
\vdots & \vdots & \vdots & \ddots & \vdots \\
\Lambda_{N} & 0 & 0 & \cdots & \beta_{N}
\end{array}\right)\left(\begin{array}{c}
\dot{X}^{e} \\
-w_{1} F_{1} \\
-w_{2} F_{2} \\
\vdots \\
-w_{N} F_{N}
\end{array}\right) .
$$

In general, the chemical potential in configuration space is of the form

$$
\mu(X, t)=k T \ln \psi(X, t)+\Phi(X) .
$$

By substitution of this equation into Eq. (36), one obtains

$$
F(X, t)=k T \frac{\partial}{\partial X}[\ln \psi(X)]+\frac{\partial \Phi(X)}{\partial X},
$$

or, expressed in terms of the initial configuration, by using Eqs. (27) and (31)

$$
F(X, t)=k T \frac{\partial}{\partial X_{0}}\left(\ln \frac{\psi_{0}\left(X_{0}\right)}{J\left(X_{0}, t\right)}\right) \cdot G^{-1}+\frac{\partial \Phi(X)}{\partial X} .
$$

This result offers the possibility to calculate the force $F$ for a given trajectory $X=\chi\left(X_{0}, t\right)$ in configuration space without a priori knowledge of the distribution function $\psi(X, t)$. In Sect. IV this will be used in numerical simulation.

An important special case of the chemical potential $\mu(X)$ is (cf. 19),

$$
\mu(X, t)=k T \ln \frac{\psi(X, t)}{\psi_{0}(X)},
$$

with $\psi_{0}$ the Boltzmann distribution,

$$
\psi_{0}(X)=\frac{1}{Z_{0}} \exp \left(-\frac{\Phi(X)}{k T}\right),
$$

and $\Phi=\Phi(X)$ a configuration dependent potential. Instead of Eq. (40) we now have

$$
F(X, t)=k T \frac{\partial}{\partial X_{0}}\left(\ln \frac{\psi_{0}\left(X_{0}\right)}{J\left(X_{0}, t\right)}\right) \cdot G^{-1}\left(X_{0}, t\right)-k T \frac{\partial}{\partial X} \ln \psi_{0}(X) .
$$

\section{A. Example: Elastic dumbbell}

In Sects. II A and II B the matrix representations of the elastic dumbbell model have been described at, respectively, the configuration tensor and the configuration space level of description. In this example, we will illustrate the Lagrangian method in more detail.

The potential $\Phi=\Phi(\mathbf{q})$ is determined, in this case, by the spring force law $\mathbf{f}^{e}=\partial \Phi / \partial \mathbf{q} \equiv \kappa(\mathbf{q}) \mathbf{q}$ in which $\kappa$ is a $\mathbf{q}$-dependent spring modulus and the equilibrium distribution (42) becomes

$$
\psi_{0}(\mathbf{q})=\frac{1}{Z_{0}} \exp \left(-\frac{\Phi(\mathbf{q})}{k T}\right) .
$$

The spring force may also be expressed as 


$$
\mathbf{f}^{e}(\mathbf{q})=-k T \frac{\partial}{\partial \mathbf{q}} \ln \psi_{0}(\mathbf{q})
$$

The Brownian force is determined by the nonequilibrium distribution function $\psi$ and is given by

$$
\mathbf{f}^{B}(\mathbf{q}, t)=-k T \frac{\partial}{\partial \mathbf{q}} \ln \psi(\mathbf{q}, t) .
$$

Contrary to the spring force, this force should not be considered as a force acting upon an individual dumbbell, but merely as an effective field acting at a given point in configuration space.

The sum of the spring force and minus the Brownian force is the thermodynamic force

$$
\mathbf{f}(\mathbf{q}, t)=\frac{\partial \mu}{\partial \mathbf{q}}=k T \frac{\partial}{\partial \mathbf{q}} \ln \frac{\psi(\mathbf{q}, t)}{\psi_{0}(\mathbf{q})} .
$$

This force is balanced by the hydrodynamic force

$$
\mathbf{f}^{h}(\mathbf{q})=-\frac{1}{2} \zeta[\dot{\mathbf{q}}(\mathbf{q}, t)-\mathbf{L}(t) \cdot \mathbf{q}]
$$

in which $\zeta$ is a friction constant and $\dot{\mathbf{q}}$ the flux velocity occurring in the continuity equation

$$
\frac{\partial \psi}{\partial t}=\frac{\partial}{\partial \mathbf{q}} \cdot(\dot{\mathbf{q}} \psi)
$$

The Lagrangian method, in this case, is based upon the mapping

$$
\mathbf{q}=\chi\left(\mathbf{q}_{0}, t\right),
$$

determined by the differential Eq. (27)

$$
\frac{\partial \chi\left(\mathbf{q}_{0}, t\right)}{\partial t}=\dot{\mathbf{q}}\left[\chi\left(\mathbf{q}_{0}, t\right), t\right]
$$

with the initial condition $\chi(\mathbf{q}, \mathbf{0})=\mathbf{q}_{0}$. The gradient of this mapping is a tensor $\mathbf{G}\left(\mathbf{q}_{0}, t\right)$, defined similar to Eq. (28) and the associated Jacobian will be denoted by $J\left(\mathbf{q}_{0}, t\right)$. Following the procedure of Sec. III we see that the distribution $\psi$ may be expressed as

$$
\psi(\mathbf{q}, t)=\frac{\psi_{0}\left(\mathbf{q}_{0}\right)}{J\left(\mathbf{q}_{0}, t\right)}
$$

and that the stress tensor $\mathbf{T}$ is obtained as

$$
\mathbf{T}=\sum_{i=1}^{N} w_{i} \mathbf{f}_{i} \mathbf{q}_{i}
$$

with $w_{i}=\psi_{0}\left(\mathbf{q}_{0 i}\right) \Delta \mathbf{q}_{0 i} / \Sigma_{i=1}{ }^{N} \psi_{0}\left(\mathbf{q}_{0 i}\right) \Delta \mathbf{q}_{0 i}$. The equation of the thermodynamic force is then given by 


$$
\mathbf{f}_{i}=k T \frac{\partial}{\partial \mathbf{q}_{0 i}}\left(\ln \frac{\psi_{0}\left(\mathbf{q}_{0 i}\right)}{J\left(\mathbf{q}_{0 i}, t\right)}\right) \cdot \mathbf{G}_{0 i}^{-1}\left(\mathbf{q}_{0 i}, t\right)-k T \frac{\partial}{\partial \mathbf{q}_{i}} \ln \psi_{0}\left(\mathbf{q}_{i}\right)
$$

In a matrix formulation analogous to Eq. (37) we now have

$$
\left(\begin{array}{c}
\mathbf{T} \\
\dot{\mathbf{q}}_{1} \\
\dot{\mathbf{q}}_{2} \\
\vdots \\
\dot{\mathbf{q}}_{N}
\end{array}\right)=\left(\begin{array}{cccll}
\mathbf{0}: & -\left(\mathbf{1} \mathbf{q}_{1}\right)^{T} \cdot & -\left(\mathbf{1} \mathbf{q}_{2}\right)^{T} \cdot & \cdots & -\left(\mathbf{1} \mathbf{q}_{N}\right)^{T} \\
\left(\mathbf{1 q}_{1}\right): & \frac{2}{\zeta w_{1}} \mathbf{1} & 0 & \cdots & 0 \\
\left(\mathbf{1} \mathbf{q}_{2}\right): & 0 & \frac{2}{\zeta w_{2}} \mathbf{1} & \cdots & 0 \\
\vdots & \vdots & \vdots & \ddots & \vdots \\
\left(\mathbf{1 q}_{N}\right): & 0 & 0 & \cdots & \frac{2}{\zeta w_{N}} \mathbf{1}
\end{array}\right)\left(\begin{array}{c}
\mathbf{L} \\
-w_{1} \mathbf{f}_{1} \\
-w_{2} \mathbf{f}_{2} \\
\vdots \\
-w_{N} \mathbf{f}_{N}
\end{array}\right) .
$$

\section{SIMULATIONS}

The matrix model is well suited for computer simulations. In the case of configuration tensor level of description, the matrix representation is of the form (7) and the algorithm consists of the calculation of the rate $\dot{\mathbf{S}}$ from the velocity gradient $\mathbf{L}$ and the thermodynamic force $\mathbf{M}(\mathbf{S})$. The value of $\mathbf{S}(t+\Delta t)$ in the next time step is approximated then as $\mathbf{S}(t)+\dot{\mathbf{S}} \Delta t$, this new value of $\mathbf{S}$ implies a new value of $\mathbf{M}$, etc. The stress tensor $\mathbf{T}$ is calculated at each time step with Eq. (7) from $\mathbf{L}$ and $\mathbf{M}$. The simulation of various types of time dependent flows in this manner turns out to be quite well possible. In Jongschaap et al. (1994), this has been demonstrated for various rheological models at the configuration tensor level of description.

At the configuration space level of description the situation is less clear. If one would use the same method for the equations listed in Sec. II B one is faced with two problems: (i) the evolution equations for $\dot{\mathbf{q}}$, $\dot{\mathbf{e}}$, etc., contain terms that depend upon the thermodynamic potential $\mu$, which in turn depends upon the distribution function $\psi$ that is not known beforehand and (ii) this distribution is also needed in the calculation of the stress tensor $\mathbf{T}$ as an average of the microscopic force dipole $\tau$.

A solution of this problem is provided by the matrix formulation (37). In that case, $N$ trajectories can be determined by solving the equations of motion for $\dot{X}_{i}$ as follows: We start with the equilibrium distribution $\psi_{0}$ which, since it is the Boltzmann distribution (42), can be obtained easily for any given model. Then, the point the point $X_{i}(\Delta t)=X_{0 i}+\dot{X}_{i} \Delta t$ is determined, where $\dot{X}_{i}$ is given by the equations of motion contained in (37) and where we have used that $F_{i}=0$ for the points $X_{0 i}$. Next, from the positions $X_{i}(\Delta t)$ one obtains the tensor $G_{i}(\Delta t)=\partial X_{i}(\Delta t) / \partial X_{0 i}$, the Jacobian $J_{i}(\Delta t)$, and thermodynamic forces $F_{i}\left(X_{i}, \Delta t\right)$ by using Eq. (43). The fluxes $\dot{X}_{i}$ are obtained then form the given $\dot{X}_{e}$, the weight factors $w_{i}$ given by (35) and the just obtained forces by using Eq. (37). In the next step one calculates in the same way $X_{i}(2 \Delta t)=X_{i}(\Delta t)+\dot{X}_{i} \Delta t, G_{i}(2 \Delta t)=\partial X_{i}(2 \Delta t) / \partial X_{i 0}, J_{i}(2 \Delta t)$, and the thermodynamic forces $F_{i}\left(X_{i}, 2 \Delta t\right)$, etc. 

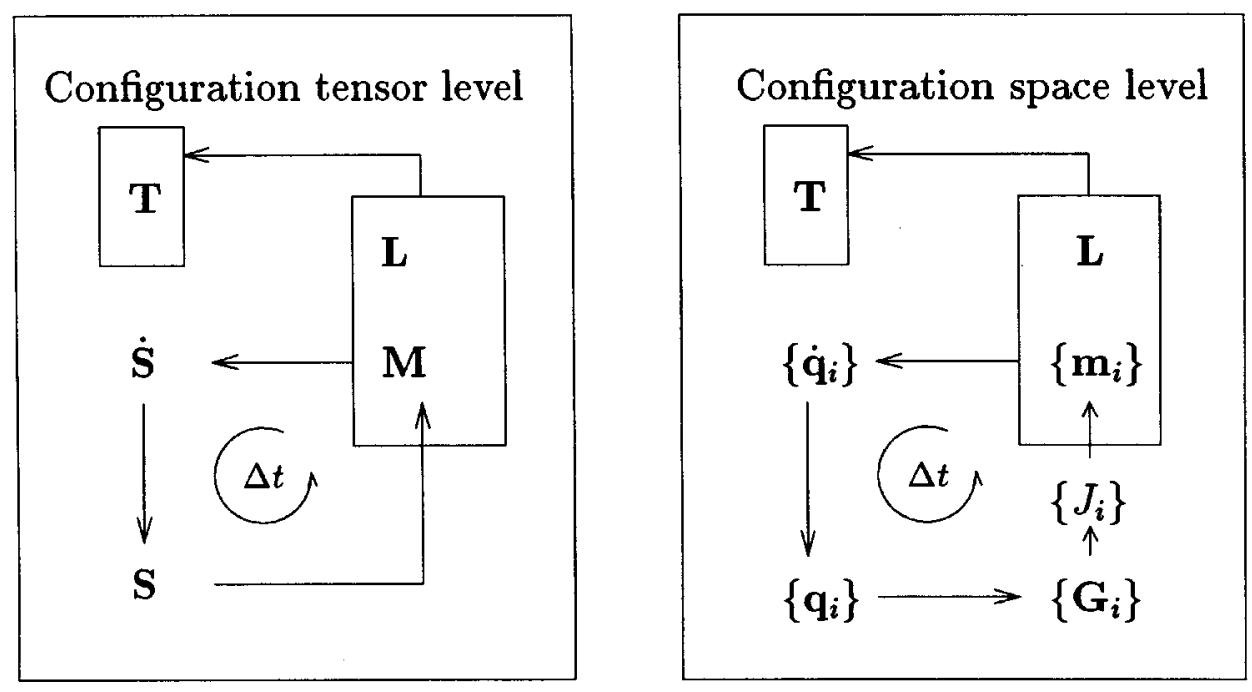

FIG. 1. Schematic representation of the algorithms based upon matrix representations at the configuration tensor and the configuration space level of description.

This algorithm, which is essentially the Lagrangian simulation method proposed by Szeri and Leal [Szeri and Leal (1992)], was used to obtain the results discussed in Sec. IV A.

The method is applicable (in principle) for any model that may be formulated in the matrix form (37) and that no a priori knowledge of the function $\chi$ is needed.

Finally, we note that the averaging needed to obtain the stress tensor is reduced to a weighted summation implied by the expression for $F^{e}$. In the next section this procedure will be discussed for the example of the elastic dumbbell model. For this case, the algorithms for the simulations at the configuration tensor and the configuration space level of description are represented schematically in Fig. 1.

\section{A. Example: Elastic dumbbell}

In the case of an elastic dumbbell model we use the matrix formulation given by Eq. (55). Starting from an arbitrary set of initial points $\left\{\mathbf{q}_{01}, \mathbf{q}_{02}, \ldots, \mathbf{q}_{0 N}\right\}$, we follow $N$ trajectories by solving the equations of motion for $\mathbf{q}_{i}$. For each time step by using Eq. (55) we obtain the value of the stress tensor $\mathbf{T}$ and also the value of the distribution function $\psi\left(\mathbf{q}_{i}, t\right)$ for each of the points $\left\{\mathbf{q}_{1}, \mathbf{q}_{2}, \ldots, \mathbf{q}_{N}\right\}$ by using Eq. (52). Figure 2 shows a typical example of the trajectory of a point, following the mapping $\mathbf{q}_{i}=\mathbf{q}\left(\mathbf{q}_{i 0}, t\right)$ for the startup of a steady shear flow, obtained in this way. After a transient, the motion follows a stationary oval orbit. Although the points $\mathbf{q}_{i}$ keep moving, the shape of the orbits becomes stationary after some time.

This result may be compared with data obtained from a Brownian dynamics simulation. To this end we performed a Brownian simulation for the same system. The stresses, obtained in both simulations show agreement within the statistical error that is demonstrated in Fig. 3. It is remarkable that whereas in the Brownian simulation at least 1000 particles are needed to obtain reliable results, in the Lagrangian simulation less than about 60 points may already be sufficient. This may even be optimized by a proper choice of the initial coordinates and by making use of symmetry properties. In Table I this is demonstrated for a steady shear flow where, because of the flow symmetry, a two- 


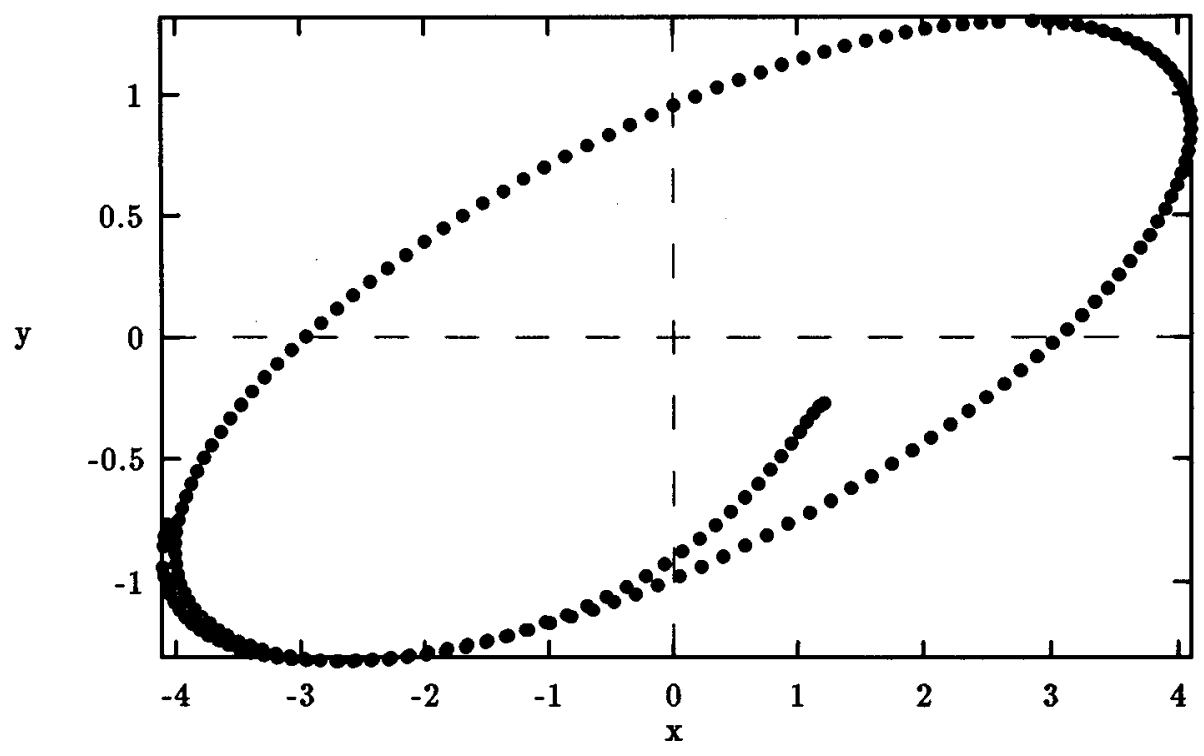

FIG. 2. Trajectory of a point in configuration space, calculated for the elastic dumbbell model at the startup of a steady shear flow. The $x$ coordinate is in the flow direction and the $y$ coordinate in the gradient direction.

dimensional simulation is sufficient in that case, and the results indicate that a number of 25 points is already sufficient to obtain accurate results. The data given in this section are intended merely as examples and a systematic study is needed before definite conclusions can be drawn. We can already say, however, that for Hookean as well as for FENE dumbbells the results obtained so far are in agreement with theoretical results and data obtained by Brownian simulations.

\section{DISCUSSION}

We have shown how the range of applicability of the matrix model, which was used previously mainly at the configuration tensor level of description, may be extended to the configuration space level of description by using the Lagrangian method proposed by Szeri and Leal. In this paper we only have outlined the principle, and for a simple

TABLE I. Relative error, in comparison with the theoretical values, in the steady shear viscosities for Hookean dumbbells obtained from the Lagrangian simulation described in Sec. IV A for various numbers of points. The results indicate that a relative small number of points is sufficient.

\begin{tabular}{cc}
\hline \hline Number of points & Error $(\%)$ \\
\hline 9 & 36 \\
16 & 4 \\
25 & 0.4 \\
36 & 0.3 \\
49 & 0.4 \\
64 & 0.4 \\
81 & 0.5 \\
100 & 0.5 \\
\hline \hline
\end{tabular}




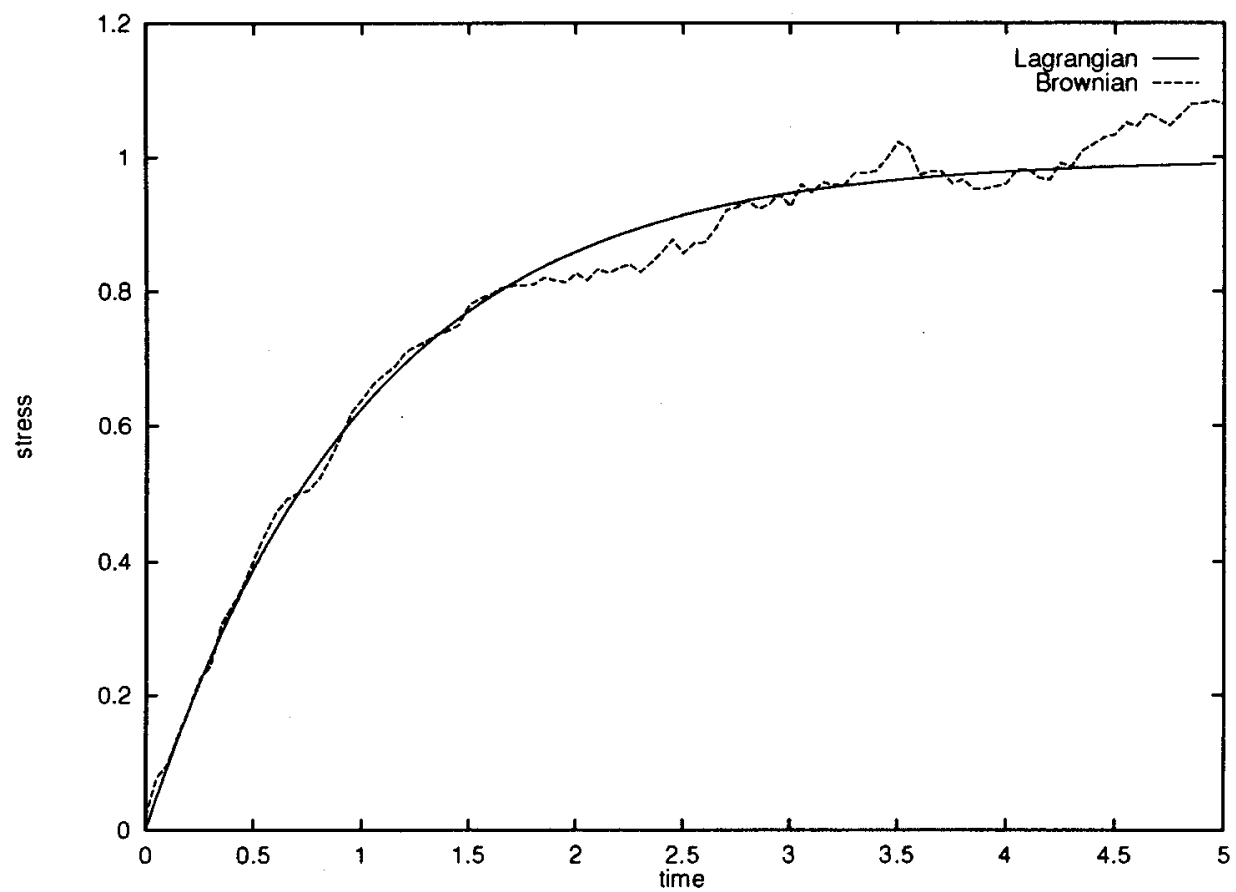

FIG. 3. Shear stresses for the startup of a steady shear flow, obtained with a Lagrangian and a Brownian simulation (time unit $\zeta / 4 \kappa$ ).

example it was demonstrated that it is possible to obtain correct results in this way. The next step will be to consider more complicated models. To this end it may be necessary to improve the efficiency of the algorithm. In the treatment of more complicated systems it will be possible to take full advantage of the matrix formulation, since it offers the possibility of consistent modifications in evolution and stress tensor expressions by a simple change of the matrix elements.

\section{ACKNOWLEDGMENTS}

The work is part of the research program of the Foundation for Fundamental Research on Matter (FOM), which is financially supported by the Netherlands Organization for Scientific Research (NWO). We also wish to thank Professor J. Mellema for helpful discussions through the course of this work.

\section{APPENDIX: EVOLUTION EQUATIONS FOR THE CONFIGURATION SPACE LEVEL OF DESCRIPTION}

In this appendix we summarize some properties of the evolution equations at the configuration space level of description. Let $X$ denote the set of configurational variables of a given system. We consider the case of continuous variables, so that the state space may be treated as a continuous manifold. Then we have a probability density

$$
\psi(X, t),
$$

normalized as 


$$
\int \psi(X, t) d X=1,
$$

which satisfies an equation of continuity

$$
\frac{\partial \psi}{\partial t}=-\frac{\partial}{\partial X} \cdot j(X, t),
$$

with a probability flux $j(X, t)$ and an associated flux velocity

$$
\dot{X}(X, t)=\frac{j(X, t)}{\psi(X, t)} .
$$

A macroscopic state is determined by a given distribution function $\psi(X, t)$. This also determines the thermodynamic state by a Gibbs' fundamental relation $U=\mathscr{F}(S,\{\psi(X, t)\})$, in which $U$ is the internal energy, $S$ the entropy, and $\mathscr{F}$ denotes the functional dependence upon $\psi(X, t)$.

For a given macroscopic (observable) state the distribution function $\psi$ is fully determined and so are the probability flux $j(X, t)$ and the flux velocity $\dot{X}(X, t)$. The state variables $X$ of a given system are stochastic and we assume that these variables are chosen such that any observable state embodies the information of the past history of the system relevant to its observational behavior. This means that the successive states form a Markov chain where the transition from a state $X$ to a state $Y$ in a time interval $\Delta t$ is determined by the transition probability $W_{X Y}$.

The configurational distribution function $\psi(X, t)$ then obeys the ChapmanKolmogorov equation

$$
\psi(Y, t+\Delta t)=\int W_{X Y}(\Delta t) \psi(X, t) d X
$$

For long times all solutions tend to a stationary solution and for a closed (no exchange of matter) and isolated (no time dependent external forces) system this stationary solution is the equilibrium state $\psi_{0}(X)$. In this state, the sum of all transitions per unit time into any state must be balanced by the sum of all transitions from that state into different states, so

$$
\int W_{X Y} \psi_{0}(X) d X=\psi_{0}(Y) \int W_{Y X} d X
$$

For closed, isolated finite physical systems under certain conditions the stronger assertion applies that for each pair of states the transitions must be balanced,

$$
W_{X Y} \psi_{0}(X)=W_{Y X} \psi_{0}(Y)
$$

This property, which under certain conditions may be derived from the invariance with respect to time reversal of the underlying microscopic equations, is called detailed balance. [This so called microscopic time reversal, should not be confused with the macroscopic time reversal used in the derivation of our matrix representation (3).]

If the state variables $X, Y, \ldots$ change only by small amounts in small time intervals, the integral equation Eq. (A5) may be expanded in a Taylor series and the so called Fokker-Planck equation is obtained, 


$$
\frac{\partial \psi}{\partial t}=-\frac{\partial}{\partial X} \cdot(C \psi)+\frac{\partial^{2}}{\partial X \partial X}:(D \psi),
$$

in which the drift function $C$ and the diffusion function $D$ are given by

$$
\begin{gathered}
C=C(X)=\lim _{\Delta t \rightarrow 0} \frac{1}{\Delta t} \int(Y-X) W_{X Y}(\Delta t) d Y, \\
D=D(X)=\lim _{\Delta t \rightarrow 0} \frac{1}{2 \Delta t} \int(Y-X)(Y-X) W_{X Y}(\Delta t) d Y .
\end{gathered}
$$

\section{Reversible and dissipative parts}

The Fokker-Planck equation (A8) can be broken up in a continuity equation

$$
\frac{\partial \psi}{\partial t}=-\frac{\partial}{\partial X} \cdot j(X, t)
$$

and a constitutive equation for the probability flux $j$,

$$
j(X, t)=C(X) \psi-\frac{\partial}{\partial X} \cdot D(X) \psi .
$$

This may also be expressed in the flux velocity $\dot{X}=j / \psi$,

$$
\dot{X}=C-\frac{1}{\psi} \frac{\partial}{\partial X} \cdot D \psi
$$

It may be proved [Kampen (1992)] that if the system obeys detailed balance, the FokkerPlanck equation has a unique decomposition into a reversible and a dissipative part,

$$
\frac{\partial \psi}{\partial t}=-\frac{\partial}{\partial X} \cdot\left(\dot{X}^{r} \psi\right)-\frac{\partial}{\partial X} \cdot\left(\dot{X}^{d} \psi\right) .
$$

The dissipative part of $\dot{X}$ determines the approach to equilibrium and is given by

$$
\dot{x}^{d}=\frac{1}{\psi_{0}} \frac{\partial}{\partial X} \cdot D \psi_{0}-\frac{1}{\psi} \frac{\partial}{\partial X} \cdot D \psi=-D \frac{\partial}{\partial X} \ln \frac{\psi}{\psi_{0}} .
$$

The reversible part of $\dot{X}$ belongs to a deterministic motion in configuration space and is given by

$$
\dot{X}^{r}=C-\frac{1}{\psi_{0}} \frac{\partial}{\partial X} \cdot D \psi_{0}
$$

For further details about this decomposition we refer to Kampen (1992).

\section{Example: Elastic dumbbell}

Let $\psi(\mathbf{q}, t)$ be the configurational distribution of elastic dumbbells with an end-to-end vector $\mathbf{q}$ in a viscous solvent. The velocity gradient of the solvent flow is $\mathbf{L}$ and the friction constant for the hydrodynamic forces on the beads is $\zeta$. The Fokker-Planck 
equation for this system is

$$
\frac{\partial \psi}{\partial t}=\underbrace{\underbrace{\frac{\partial}{\partial \mathbf{q}} \cdot(\psi \mathbf{L} \cdot \mathbf{q})}_{\text {Spring force }}-\underbrace{\frac{\partial}{\partial \mathbf{q}} \cdot\left(\psi \frac{2 k T}{\zeta} \frac{\partial}{\partial \mathbf{q}} \ln \psi_{0}\right)}_{\text {Diffusion }}}_{\text {Convection }}+\underbrace{\frac{\partial}{\zeta}}_{\frac{\partial}{\partial \mathbf{q}} \cdot\left(\psi \frac{2 k T}{\zeta} \frac{\partial}{\partial \mathbf{q}} \ln \psi\right)}
$$

In this case we have

$$
C=\mathbf{L} \cdot \mathbf{q}+\frac{2 k T}{\zeta} \frac{\partial}{\partial \mathbf{q}} \ln \psi_{0}
$$

and

$$
D=\frac{2 k T}{\zeta} \mathbf{1}
$$

The flux $\dot{\mathbf{q}}$ becomes

$$
\dot{\mathbf{q}}=\mathbf{L} \cdot \mathbf{q}+\frac{2 k T}{\zeta} \frac{\partial}{\partial \mathbf{q}} \ln \psi_{0}-\frac{2 k T}{\zeta \psi} \frac{\partial}{\partial \mathbf{q}} \psi=\mathbf{L} \cdot \mathbf{q}-\frac{2 k T}{\zeta} \frac{\partial}{\partial \mathbf{q}}\left(\ln \frac{\psi}{\psi_{0}}\right),
$$

with a reversible part

$$
\begin{gathered}
\dot{\mathbf{q}}^{r}=C-\frac{1}{\psi_{0}} \frac{\partial}{\partial \mathbf{q}} \cdot D \psi_{0}, \\
=\mathbf{L} \cdot \mathbf{q}
\end{gathered}
$$

and a dissipative part

$$
\dot{\mathbf{q}}^{d}=-\frac{2 k T}{\zeta} \frac{\partial}{\partial \mathbf{q}} \ln \frac{\psi}{\psi_{0}},
$$

so the representation of the Fokker-Planck equation analogous to Eq. (A13) becomes

$$
\frac{\partial \psi}{\partial t}=\underbrace{-\frac{\partial}{\partial \mathbf{q}} \cdot(\psi \mathbf{L} \cdot \mathbf{q})}_{\text {Reversible Part }}+\underbrace{\frac{\partial}{\partial \mathbf{q}} \cdot\left(\psi \frac{2 k T}{\zeta} \frac{\partial}{\partial \mathbf{q}} \ln \frac{\psi}{\psi_{0}}\right)}_{\text {Dissipative Part }} .
$$

Note that the decomposition obtained in this way is consistent with the decomposition in the evolution equation for $\dot{\mathbf{q}}$ implied by the matrix formulation (18).

\section{References}

Beris, A. and B. Edwards, The Thermodynamics of Flowing Systems (Oxford University Press, New York, 1994).

Curtiss, C. F. and R. B. Bird, "Kinetic theory for polymer melts. I. The equation for the single-link orientation distribution function," J. Chem. Phys. 75, 2016-2033 (1981).

Doi, M., "Explanation of the 3.4 power law of viscosity on the basis of a tube model," J. Polym. Sci. Phys. Ed. 19, 667-684 (1981). 
Giesekus, H., "A simple constitutive equation for polymer fluids, based upon the concept of a deformation dependent tensorial mobility," J. Non-Newt. Fluid Mech. 11, 69-109 (1982a).

Giesekus, H., "A unified approach to a variety of constitutive models for polymer fluids, based upon the concept of a configuration dependent molecular mobility,' Rheol. Acta 21, 366-375 (1982b).

Grmela, M., "Bracket formulation of diffusion-convection equations," Physica D 21, 179-212 (1986).

Groot, S. and P. Mazur, Non-equilibrium Thermodynamics (North-Holland, Amsterdam, 1969).

Jongschaap, R., "'Microscopic modeling of the flow properties of polymers,'” Rep. Prog. Phys. 53, 1-55 (1990).

Jongschaap, R., "Towards a unified formulation of microrheological models," Springer Lecture Notes in Physics 381, 215-247 (1991).

Jongschaap, R., K. de Haas, and C. Damen, “A generic matrix representation of configuration tensor rheological models,” J. Rheol. 38, 769-795 (1994).

Jongschaap, R., "Some comments on reptation theories," Rheol. Acta 26, 99-102 (1988).

Kampen, N. V., Stochastic Processes in Physics and Chemistry (North Holland, Amsterdam, 1992).

Kluitenberg, G., "Thermodynamic theory of elasticity and plasticity," Physica 28, 217-232 (1962).

Larson, R., “A constitutive equation for polymer melts, based upon partially extending convection,'” J. Rheol. 28, 545-571 (1984).

Leonov, A., "Non-equilibrium thermodynamics and rheology of viscoelastic polymer media,' Rheol. Acta 15, 85-98 (1976).

Lhuillier, D. and A. Ouibrahim, “A thermodynamic model for solutions of deformable particles," J. Mech. 19, 725-741 (1980).

Martin, P., O. Parodi, and P. Pershan, "Unified hydrodynamic theory for liquid crystals, and normal fluids," Phys. Rev. A 6, 2401-2420 (1972).

Maugin, G. and R. Drouot, "Internal variables and the thermodynamics of macromolecule solutions,' Int. J. Eng. Sci. 21, 705-724 (1983).

Meixner, J., "Die thermodynamische theorie der relaxationserscheinungen und ihr zusammenhang mit der nachwirkungstheorie,' Kolloid-Zeitschrift 134, 3-19 (1953).

Schieber, J. and H. Öttinger, "On the consistency criteria for stress tensors in kinetic theory models," J. Rheol. 38, 1909-1924 (1994).

Szeri, A. and L. Leal, "A new computational method for the solution of flow problems of microstructured fluids, Part I. Theory,” J. Fluid Mech. 242, 549-576 (1992).

Tervoort, T. A., Constitutive Modeling of Polymer Glasses, Ph.D. thesis, University of Eindhoven (1996). 